\title{
Film based verification of calculation algorithms used for brachytherapy planning-getting ready for upcoming challenges of MBDCA
}

\author{
Grzegorz Zwierzchowski, MSc, PhD',2, Grzegorz Bielęda, MSc², Prof. Janusz Skowronek, MD, PhD',3, Magdalena Mazur, MSc \\ 'Department of Electroradiology, Faculty of Health Sciences, Poznan University of Medical Sciences, ${ }^{2}$ Medical Physics Department, Greater Poland \\ Cancer Centre, ${ }^{3}$ Brachytherapy Department, Greater Poland Cancer Centre, ${ }^{4}$ Medical Physics Department, Adam Mickiewicz University, Poznan, Poland
}

\begin{abstract}
Purpose: Well-known defect of TG-43 based algorithms used in brachytherapy is a lack of information about interaction cross-sections, which are determined not only by electron density but also by atomic number. TG-186 recommendations with using of MBDCA (model-based dose calculation algorithm), accurate tissues segmentation, and the structure's elemental composition continue to create difficulties in brachytherapy dosimetry. For the clinical use of new algorithms, it is necessary to introduce reliable and repeatable methods of treatment planning systems (TPS) verification. The aim of this study is the verification of calculation algorithm used in TPS for shielded vaginal applicators as well as developing verification procedures for current and further use, based on the film dosimetry method.

Material and methods: Calibration data was collected by separately irradiating 14 sheets of Gafchromic ${ }^{\circledR}$ EBT films with the doses from $0.25 \mathrm{~Gy}$ to $8.0 \mathrm{~Gy}$ using HDR ${ }^{192} \mathrm{Ir}$ source. Standard vaginal cylinders of three diameters were used in the water phantom. Measurements were performed without any shields and with three shields combination. Gamma analyses were performed using the VeriSoft ${ }^{\circledR}$ package.

Results: Calibration curve was determined as third-degree polynomial type. For all used diameters of unshielded cylinder and for all shields combinations, Gamma analysis were performed and showed that over $90 \%$ of analyzed points meets Gamma criteria ( $3 \%, 3 \mathrm{~mm})$.

Conclusions: Gamma analysis showed good agreement between dose distributions calculated using TPS and measured by Gafchromic films, thus showing the viability of using film dosimetry in brachytherapy.

Key words: brachytherapy, film dosimetry, QA, Gamma analysis, shields.

\section{Purpose}

Designing and introducing reliable methods of verification treatment planning systems (TPS) used in brachytherapy is a complex project in most cases $[1,2,3]$. The TPS used in most of the cases are still based on the TG-43 recommendations, which have been verified in homogenous conditions [4]. However, one of the wellknown defects of the calculation algorithms based on the Sivert integral and modular dose calculation models is that such dose rate calculations are based on a single source position in a homogenous water environment $[5,6,7]$, whereas the overall dose distribution in the medium is a product of the contribution from each source position and the modulated step time, which are governed by optimization routines $[8,9]$.

Three-dimensional (3D) reconstruction of the geometry is a common technique $[10,11,12]$ but the benefits from us- ing these imaging methods are not fully realized because of the lack of usable information about the cross-sections interaction, which are determined not only by electron density but also by the atomic number $(\mathrm{Z})$ of the applicator itself and surrounding tissues [13]. As a result, for the relatively low energy range commonly used in brachytherapy, it is impossible to determine the influence of heterogeneities on dose calculations. TG-186 recommendations provide greater accuracy in brachytherapy dosimetry. The TG-186 recommends the use of the MBDCA (model-based dose calculation algorithm), and emphasizes the need for accurate tissue segmentation by identifying the tissue type in terms of density and elemental composition $[14,15,16]$. However, for the clinical use of these new calculation methods, reliable and repeatable methods of TPS verification are needed.

Large share of recent studies is focused on Monte Carlo (MC) simulations, which are independent of accuracy of manual setup of experiment $[17,18,19]$. Many facilities 

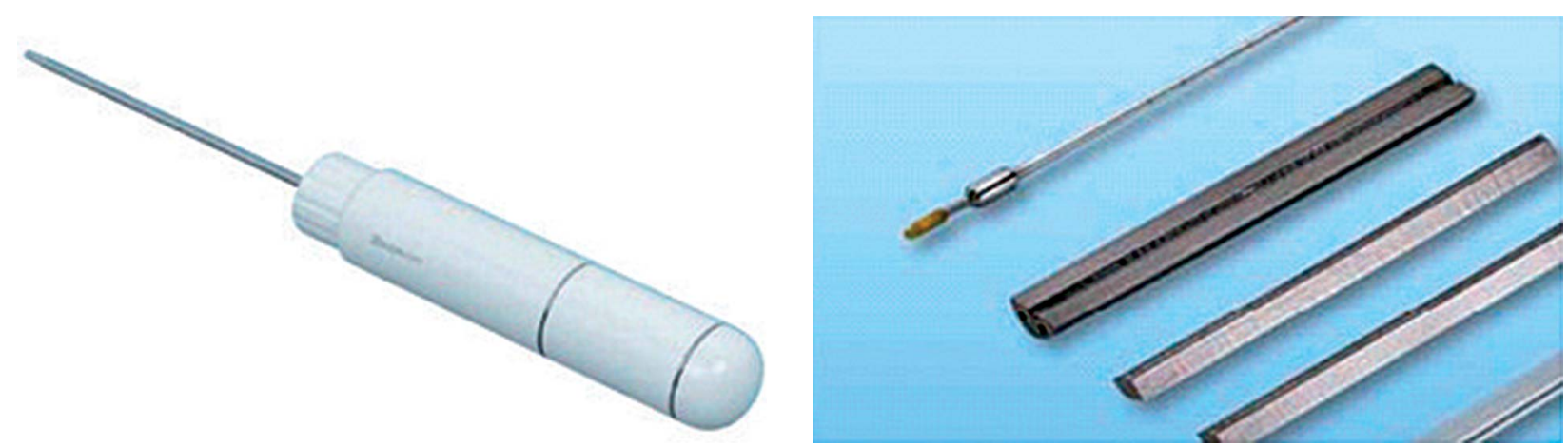

Fig. 1. Standard vaginal cylinder with a set of interchangeable shields (Elekta Brachy ${ }^{\circledR}$ )

do not perform MC simulations and are dependent on different verification methods. Self-developing dosimetry films have been used to verify dose distributions, and their use has been well documented [20,21,22,23]. However, they need to be adapted to the technical capacities of the particular facility [24]. Our department is preparing to introduce MBDCA soon. For this reason, a setup was prepared, which was verified using algorithms based still on TG-43 recommendations. For this reason, a common brachytherapy setup was used, involving vaginal cylinders and internal shields as a test platform to develop a verification procedure for reaffirmation of the MBDCA based algorithms recommended in TG-186, which will be used after implementation of MBDCA.

The aim of this study is to develop dose distribution verification procedure based on film dosimetry, which may be easily introduced in brachytherapy department.

\section{Material and methods}

\section{Film calibration}

Calibration data were collected by separately irradiating 14 sheets $\left(20 \mathrm{~mm} \times 30 \mathrm{~mm}\right.$ ) of Gafchromic ${ }^{\circledR}$ EBT (Lot \#: 47207-031, ISP, Ashland, Covington, USA) films with doses ranging from $0.25 \mathrm{~Gy}$ to $8.0 \mathrm{~Gy}$, using HDR ${ }^{192} \mathrm{Ir}$ (192Ir-mHDR-v2, Nucletron, an Elekta company, Elekta $\mathrm{AB}$, Stockholm, Sweden) source. To assure homogenous dose distribution, films were placed between two blocks of $25 \mathrm{~mm}$ thick PMMA, and two catheters were located above and below that films at a distance of $25 \mathrm{~mm}$. Doses were prescribed to the dose points in the center of a film. After 72 hours, the films were digitized with a flat table scanner (Epson ${ }^{\circledR}$ Perfection V750 Pro, Seiko Epson Corporation, Suwa, Japan) with light source on the one side and the detector on the other side of the film, all with the same orientation. Mean values from the most homogenous central part of the film $(10 \mathrm{~mm} \times 5 \mathrm{~mm})$ were calculated using the VeriSoft ${ }^{\circledR}$ (PTW, Freiburg GMBH, Freiburg, Germany) package. In region of interest of $10 \mathrm{~mm} \times 5 \mathrm{~mm}$, the dose variation was estimated below $5 \%$. The calibration curve and calculation of the optical density to the doses was prepared (analog to digital conversion value; ADC).

\section{Verification of the dose distribution}

Standard vaginal cylinders (Nucletron, an Elekta company, Elekta AB, Stockholm, Sweden) with three different diameters $(25,35$, and $40 \mathrm{~mm})$ were used as applicators (Figure 1). Oncentra Brachy 4.3.1 with implemented applicator modelling, including shielding for gynecological applicators was applied.

Dosimetric media (Gafchromic ${ }^{\circledR}$ EBT dosimetric films) were prepared by cutting a round hole in the center of the sheets to fit precisely to the diameter of the cylinders. The OLFA ${ }^{\circledR}$ Circle Cutter (Olfa Corporation, Osaka, Japan) was used to assure sharp, clean edges of the cut, in order to minimize water penetration into the layered structure of the film. Film sheets were placed perpendicular to the axis of the applicator, in the position corresponding to the center of the planned active length of $25 \mathrm{~mm}$ (6 active positions of the source) (Figure 2). The computed tomography $(\mathrm{CT})$ of the cylinder was performed with markers on surface of the cylinder to ensure the plane in which the film was to be placed. OncentraBrachy ${ }^{\circledR} 4.3 .1$ (TG-43) (Nucletron, an Elekta company, Elekta AB, Stockholm, Sweden) was used to calculate the dose distribution.

The applicator was then submersed in the water phantom $(50 \times 50 \times 50 \mathrm{~cm})$. Measurements were performed for all three cylinder diameters without using any shields, and separately, with a combination of three shields. Three shielding angles $\left(90^{\circ}, 180^{\circ}\right.$, and $\left.270^{\circ}\right)$ were used (Figure 3). After 72 hours, the films were digitized. Scanning was performed on Epson Perfection V750 Pro scanner (Seiko Epson Corporation, Suwa, Japan) with 96 dpi resolution and 48 bit colour depth in red light colour spectrum.

Gamma analysis of the data was performed using the VeriSoft $^{\circledR}$ (PTW, Freiburg GMBH, Freiburg, Germany) package. The dose distributions calculated by the TPS were

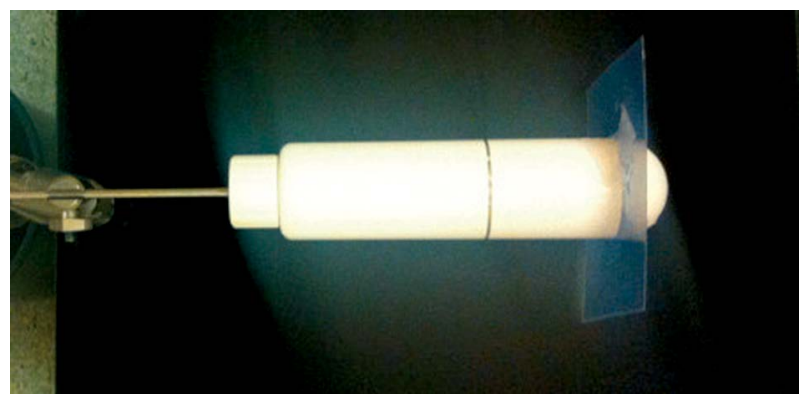

Fig. 2. Measurement setup with dosimetric film fixed perpendicular to the applicator, ready for submersion in the water phantom 

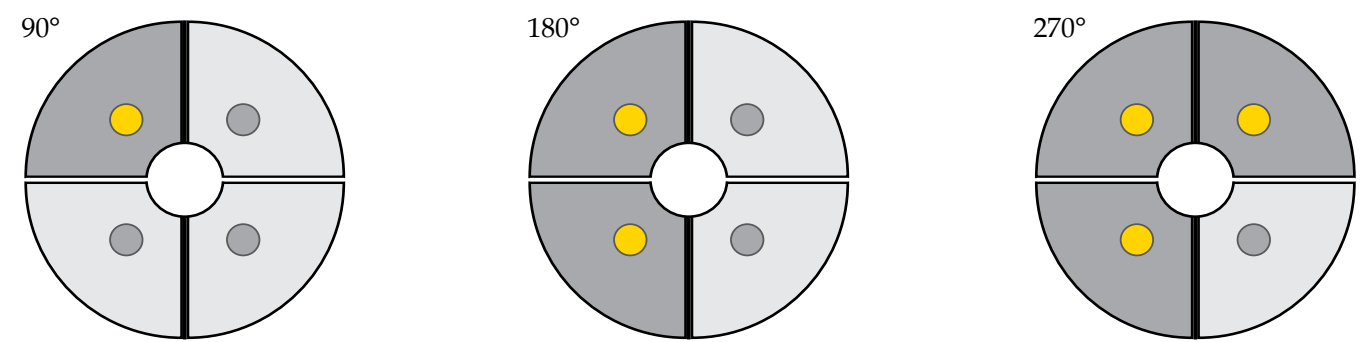

Fig. 3. Cross-section of three different shields combinations for $90^{\circ}, 180^{\circ}$, and $270^{\circ}$ shielding angle (yellow dot presents the shielding)

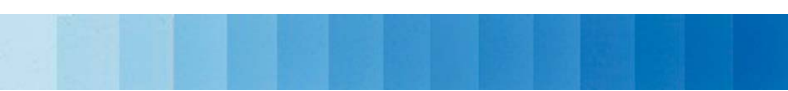

Fig. 4. Digitized sheets of the films used to obtain calibration data. Deposited doses from left to right are: $0.0 \mathrm{~Gy}$, $0.25 \mathrm{~Gy}$, and 0.5-5.0 Gy with 0.5 Gy intervals, and 5-8 Gy with 1.0 Gy intervals

prepared as dose grids with a planar resolution of $1.0 \mathrm{~mm}$. Data packages obtained in this process were imported into the VeriSoft ${ }^{\circledR}$ software and compared to the data obtained from Gafchromic EBT films. As there are no general rules for Gamma analysis in brachytherapy dosimetry, we used the AAPM (The American Association of Physicists in Medicine) TG-119 recommendations for Gamma analysis in IMRT ( $3 \%$ and $3 \mathrm{~mm}$, with a level of $90 \%$ for acceptance). Analyses were performed for the treatment plans with and without shields, and for the various cylinder diameters $25 \mathrm{~mm}, 35 \mathrm{~mm}$, and $40 \mathrm{~mm}$. Three shields combinations were used: $90^{\circ}, 180^{\circ}$, and $270^{\circ}$, respectively.

\section{Results}

\section{Calibration data}

To determine the calibration data, 14 sheets of films were irradiated separately using the PMMA phantom, as described previously. Figure 4 shows the darkening

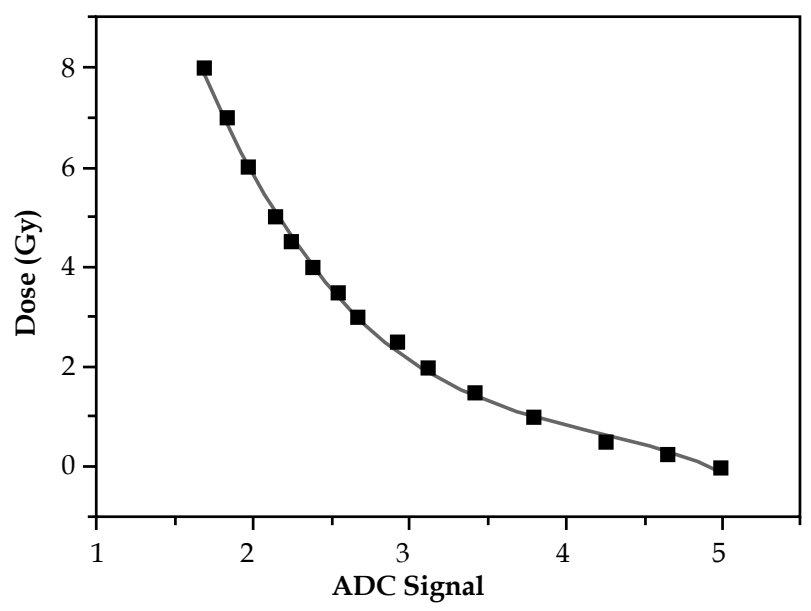

Fig. 5. Calibration curve for the Gafchromic EBT films and ${ }^{192}$ Ir source

(bluing) of the film for doses used during the calibration process. Standard deviation of the measured ADC values was $0.82 \%$.

The calibration curve (Figure 5) was determined as the third degree polynomial type:

$$
y=A_{0}+A_{1} x^{1}+A_{2} x^{2}+A_{3} x^{3},
$$

where: $\mathrm{A}_{0}=29.02, \mathrm{~A}_{1}=-18.89, \mathrm{~A}_{2}=4.35, \mathrm{~A}_{3}=-0.35$.
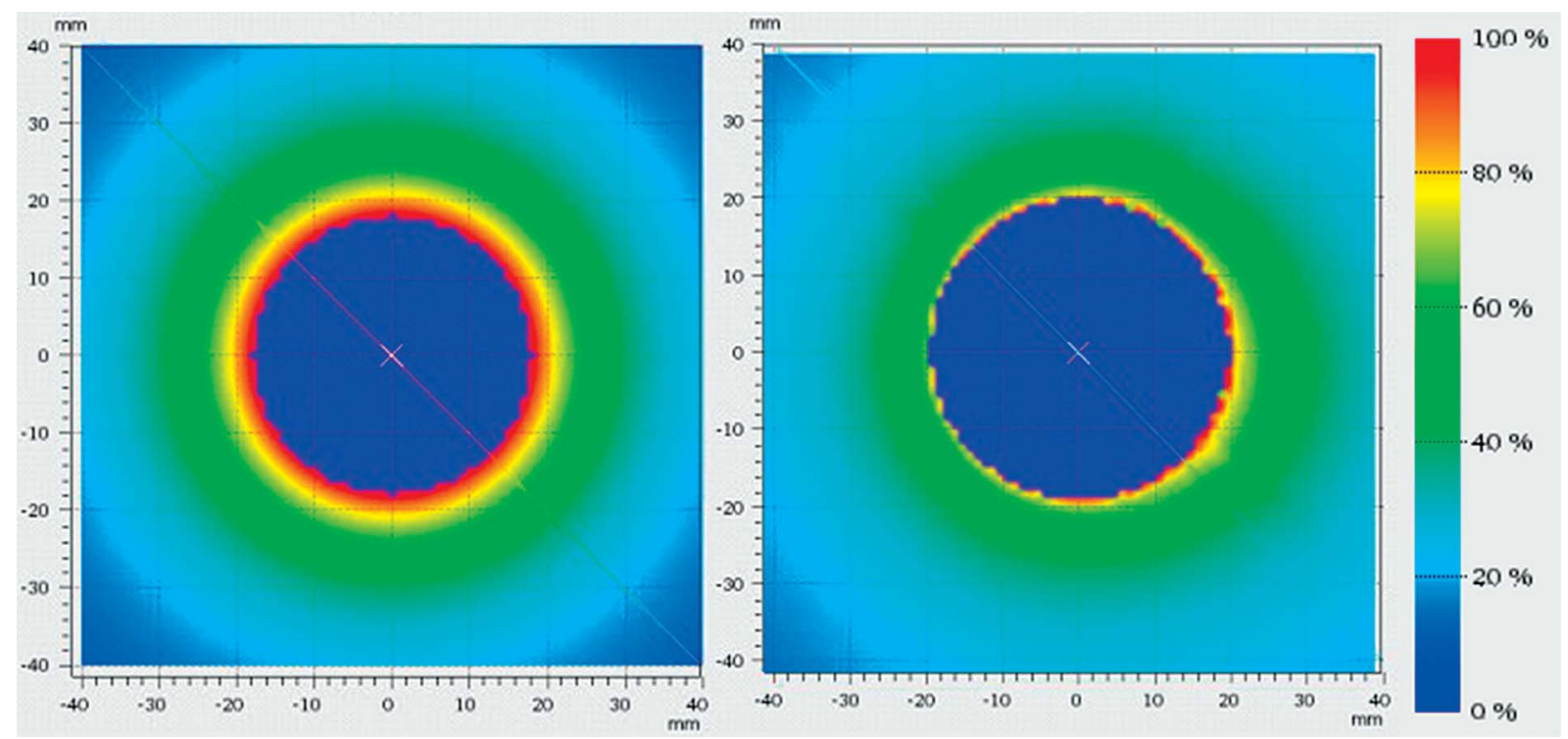

Fig. 6. Dose distribution for the $35 \mathrm{~mm}$ unshielded cylinder $(100 \%=6.024 \mathrm{~Gy})$. On the left side is the calculated dose (TPS), and on the right side, the film-measured data 
The goodness of fit of calculated polynomial: $r_{a}^{2}=$ 0.99725 .

\section{Dosimetric verification of the dose distribution using Gamma method}

Co-registration of data imported from TPS and dose distribution from scanned film were guided by the center of cylinder. Rotation of the film was corrected in shielded applicator by seeking the steepest dose gradient in the shielded/unshielded area, and was performed manually. Dose was normalized to $100 \%$ as maximum measured dose by VeriSoft ${ }^{\circledR}$ software for each analyzed film. Graphical representation of the Gamma values obtained for $35 \mathrm{~mm}$ cylinders is presented as an example in Figure 6 to Figure 13. Dose distribution was not normalized. The Figures are presentation of calculated dose distribution in TPS and ADC obtained from scanned Gafchromic films. For the graphical representation of the Gamma values, green and blue shades represent agreement at 10095\% level, yellow at $95-90 \%$, and red below $90 \%$.

Tables 1, 2, and 3 provide summarized data of the Gamma analysis for the three different cylinder diameters, both unshielded, and with the three shield combinations. Number of analyzed points varied due to film rotation in software (the rotation closer to $45^{\circ}$, additional data was lost).

In all the cases, Gamma analysis showed good agreement between the dose distribution calculated with TG-43
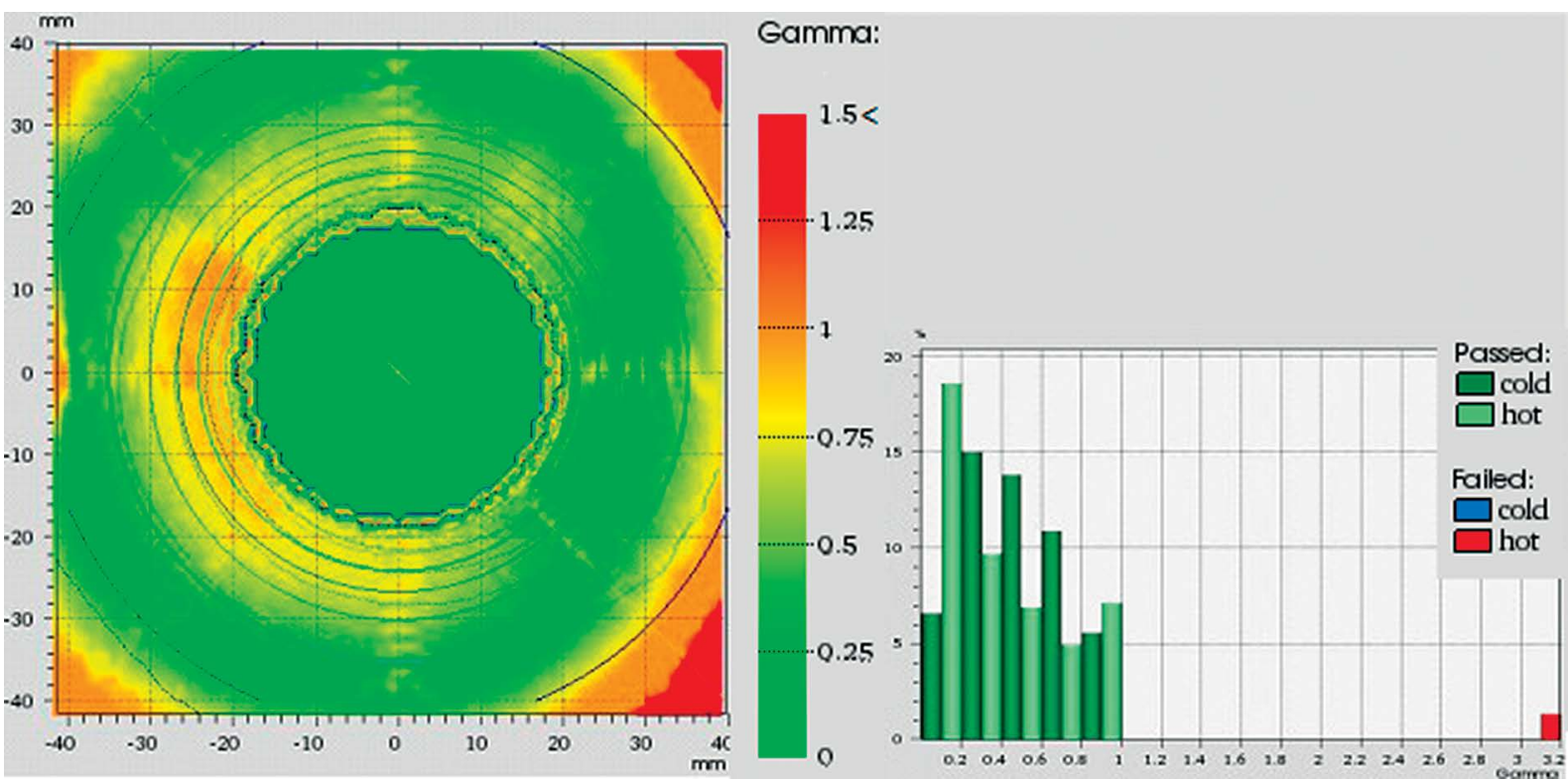

Fig. 7. Gamma values for the $35 \mathrm{~mm}$ unshielded cylinder. The left side shows the graphical representation (see text), and the right side shows the Gamma values histogram
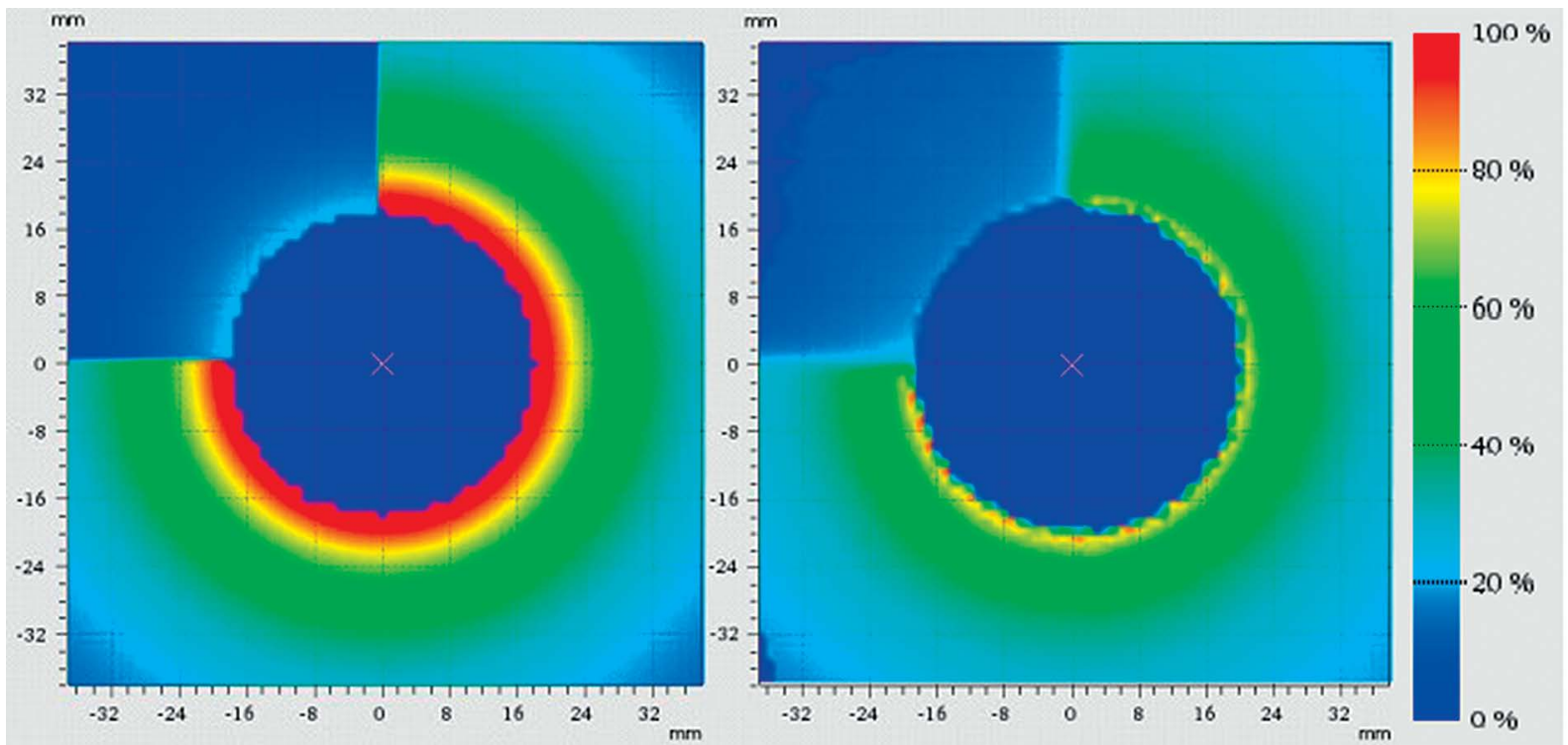

Fig. 8. Dose distribution for the $35 \mathrm{~mm}$ cylinder with $90^{\circ}$ shielding $(100 \%=6.155 \mathrm{~Gy})$. On the left side is the calculated dose (TPS), and on the right side, the film-measured data 

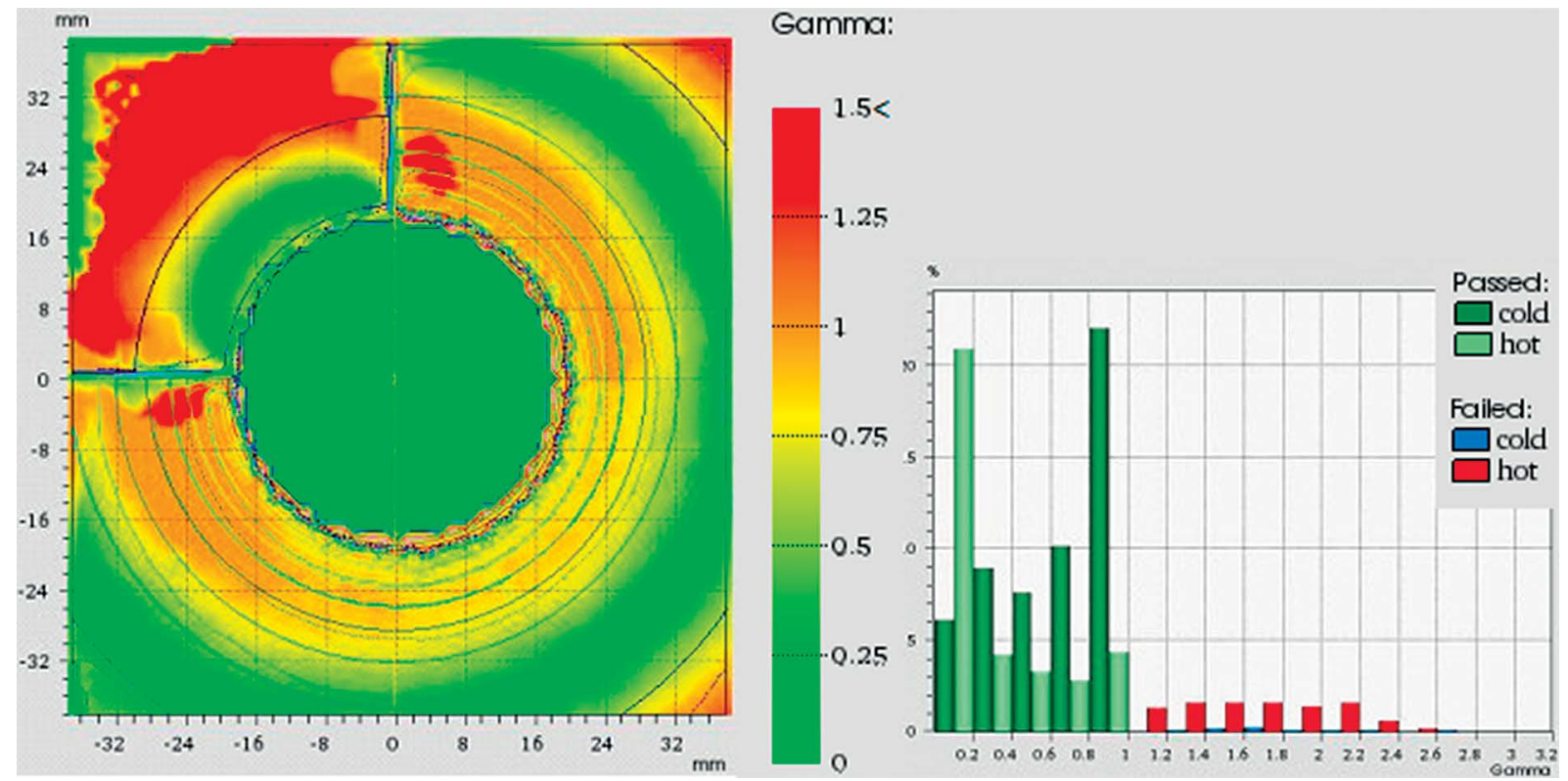

Fig. 9. Gamma values for the $35 \mathrm{~mm}$ cylinder with $90^{\circ}$ shielding. On the left side is the graphical representation (see text), and on the right side, the Gamma values histogram
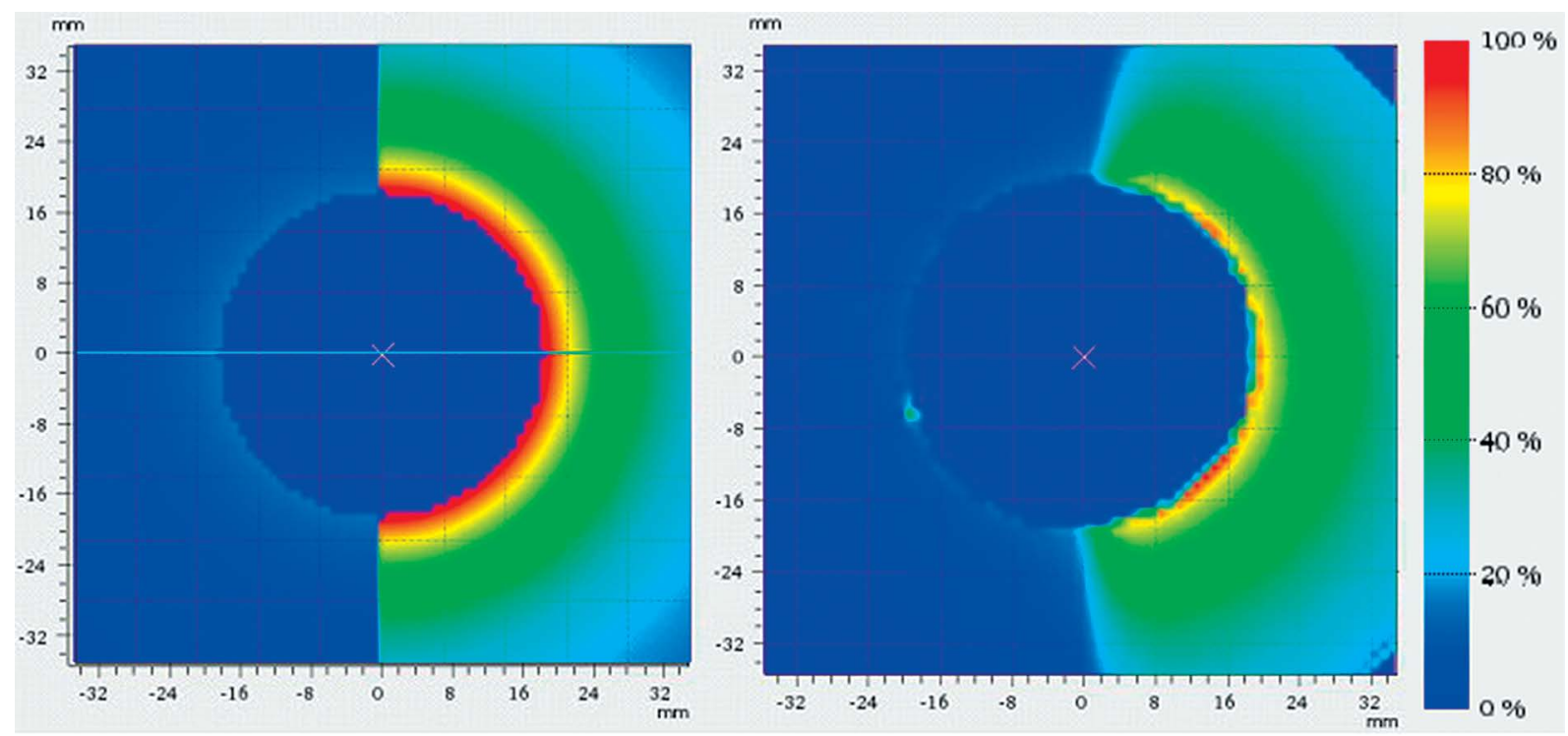

Fig. 10. Dose distribution for the $35 \mathrm{~mm}$ cylinder with $180^{\circ}$ shield $(100 \%=6.029 \mathrm{~Gy})$. On the left side is the calculated dose (TPS), and on the right side, the film-measured data

based TPS and measured with Gafchromic ${ }^{\circledR}$ films. For all diameters of unshielded cylinder and for all shield combinations, Gamma analyses showed that over $90 \%$ of the analyzed points met Gamma criteria ( $3 \%, 3 \mathrm{~mm})$.

\section{Discussion}

In this study, we have attempted to use the typical brachytherapy setup with vaginal cylinders and internal shields as a test platform to develop a quality assurance procedure, verifying the calculation algorithm implemented to TPS. The main finding of our study was that self-developing flat film dosimetry is a reliable verification method that could be easily adapted to almost any clinical setup where point dose dosimetry is difficult to use, and cannot provide valuable information.

\section{Calibration}

Many Gafchromic EBT film detector calibration methods are available. One such method involves the use of externally generated photon beams, which was the easiest calibration method to implement. However, even though this calibration method was common [25,26,27], its use has been abandoned. Chiu-Tsao et al. [28] demonstrated that the films response to irradiation with $21 \mathrm{keV}$ and $6 \mathrm{MV}$ photon beams is practically independent of the energy, but is notably worse at low energies. Similar 

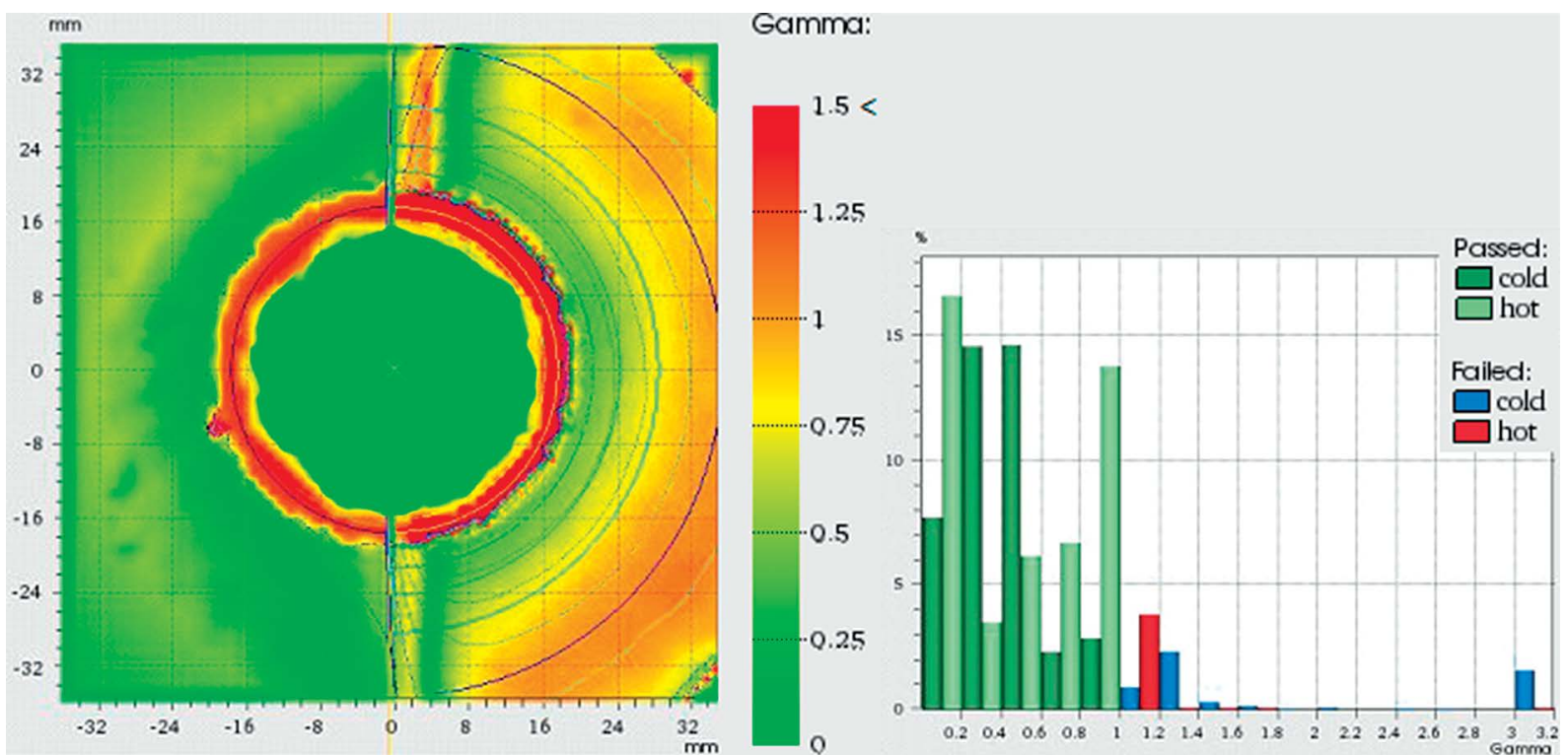

Fig. 11. G Gamma values for the $35 \mathrm{~mm}$ cylinder with $180^{\circ}$ shielding. On the left side is the graphical representation (see text), and on the right side, the Gamma values histogram
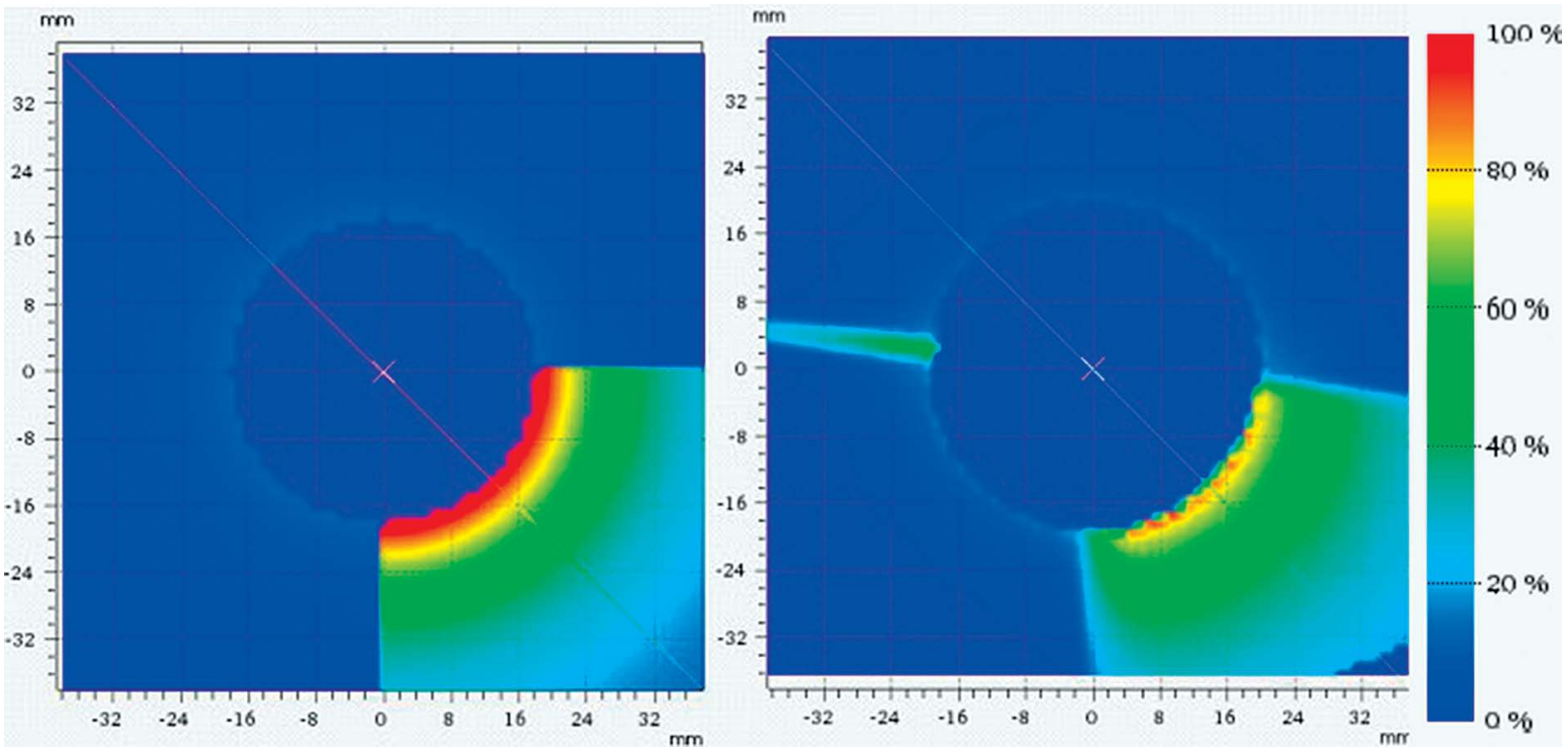

Fig. 12. Dose distribution for the $35 \mathrm{~mm}$ cylinder with $270^{\circ}$ shield $(100 \%=5.804 \mathrm{~Gy})$. On the left side is the calculated dose (TPS), and on the right side, the film-measured data, with leakage clearly visible

results for EBT films were demonstrated by Butson et al. [29] for the $50 \mathrm{keV}-6 \mathrm{MV}$ energy range. However, Brown and Hongstrom [27], irradiated EBT films up to $3 \mathrm{~Gy}$ with beams of energies varying between $25 \mathrm{keV}$ and $4 \mathrm{MV}$, and proved that films were sensitive to the beam energy. Richter reached a similar conclusion [30]. These contradictory results may be due to varying reasons. Lack of unanimous results, which would support definitely the statistical insignificance of the dependence of radiochromic film's reaction to radiation energy (especially lower than $500 \mathrm{kV}$ ), convinced us to use iridium source for film calibration. Homogenous irradiation of a piece of film detector with a point source in one position [31] is not achievable, therefore, a special arrangement of irradiation based on Khushdeep Singh's [21] work with two catheters was used. That allowed to deposit not less than $95 \%$ with standard deviation of $0.82 \%$ of reference dose on a defined film area.

\section{Dose distribution analysis}

After calibration of the Gafchromic films using a phantom, and digitization of irradiated films, the data for analysis was obtained. The software-converted data sets were compared to digital data imported from the TPS using a 2D gamma comparison in Verisoft ${ }^{\circledR}$, a software package, which is usually used for dosimetric analysis 

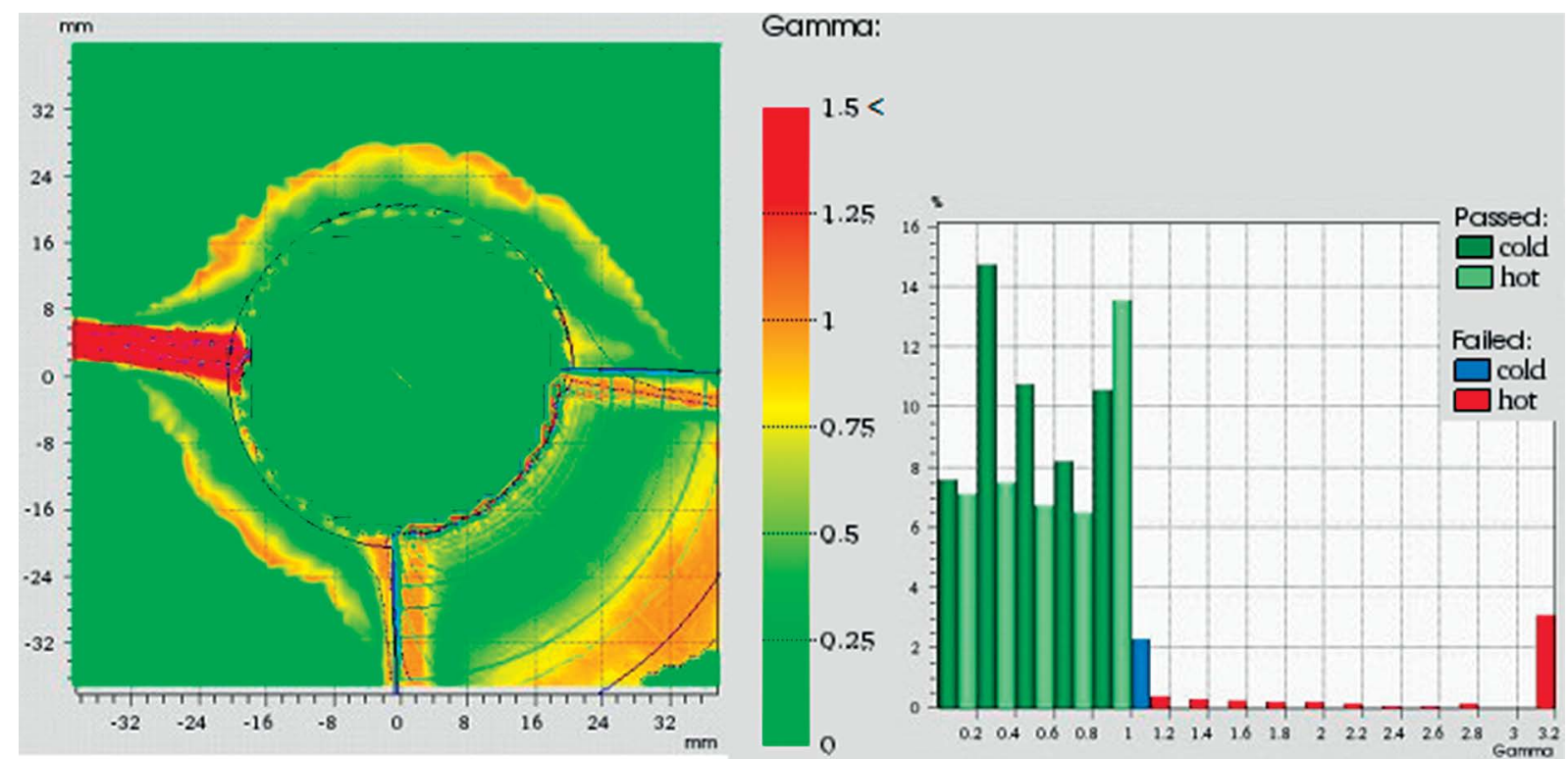

Fig. 13. Gamma values for the $35 \mathrm{~mm}$ cylinder with $270^{\circ}$ shield. On the left is the graphical representation (see text). The image on the right shows the Gamma value histogram with leakage clearly visible

Table 1. Summarized Gamma parameters for $25 \mathrm{~mm}$ diameter cylinder

\begin{tabular}{lcccc}
\hline 2D Gamma parameters $(25 \mathrm{~mm})$ & Shield $0^{\circ}$ (none) & Shield $90^{\circ}$ & Shield $180^{\circ}$ & Shield $270^{\circ}$ \\
\hline No. of analyzed points & 6562 & 6561 & 5041 & 6561 \\
\hline Points meeting Gamma criteria [\%] & $\mathbf{9 5 . 2}$ & $\mathbf{9 1 . 0}$ & $\mathbf{8 9 . 1}$ & $\mathbf{9 2 . 2}$ \\
\hline \begin{tabular}{l} 
2D Gamma values \\
\hline Mean
\end{tabular} & 0.735 & & & 0.706 \\
\hline Minimum & 0.000 & 0.841 & 5.216 & 0.001 \\
\hline Maximum & 16.126 & 16.625 & 18.665 & 1.803 \\
\hline Median & 0.363 & 0.521 & 0.711 & 0.726
\end{tabular}

Table 2. Summarized Gamma parameters for $35 \mathrm{~mm}$ diameter cylinder

\begin{tabular}{lcccc}
\hline 2D Gamma parameters $(35 \mathrm{~mm})$ & Shield $0^{\circ}$ (none) & Shield $90^{\circ}$ & Shield $180^{\circ}$ & Shield $270^{\circ}$ \\
\hline No. of analyzed points & 6561 & 5852 & 5041 & 5929 \\
\hline Points meeting Gamma criteria [\%] & $\mathbf{9 8 . 7}$ & $\mathbf{9 0 . 0}$ & $\mathbf{9 2 . 6}$ & $\mathbf{9 3 . 1}$ \\
\hline 2D Gamma values & & & 0.650 & 0.790 \\
\hline Mean & 0.521 & 0.610 & 0.000 & 0.000 \\
\hline Minimum & 0.000 & 0.000 & 7.026 & 15.728 \\
\hline Maximum & 12.323 & 2.740 & 0.466 & 0.548
\end{tabular}

in IMRT. As there is no general rule for Gamma analysis in brachytherapy dosimetry, we used the AAPM TG-119 recommendations for Gamma analysis in IMRT ( $3 \%$ and $3 \mathrm{~mm}$, with a level of $90 \%$ for acceptance). Although this range is the most popular, it is not the only one used in common practice. The criterion value depends on the type of analysis and required acceptance level in the established area. The gamma comparison of 12 films was acceptable in 11 cases at the $90 \%$ acceptance level. The highest level of acceptance was achieved for all the applicator diameters not filled with tungsten shields. This confirms the accuracy of the measurements and encourages for further analysis when shields are included. These findings also acknowledge that the TG-43 
Table 3. Summarized Gamma parameters for $40 \mathrm{~mm}$ diameter cylinder

\begin{tabular}{lcccc}
\hline 2D Gamma parameters $(40 \mathrm{~mm})$ & Shield $0^{\circ}$ (none) & Shield $90^{\circ}$ & Shield $180^{\circ}$ & Shield $270^{\circ}$ \\
\hline No. of analyzed points & 6561 & 6561 & 5041 & 5504 \\
\hline Points meeting Gamma criteria [\%] & 95.1 & 90.3 & 91.4 & 90.7 \\
\hline 2D Gamma values & & & 0.821 & 0.902 \\
\hline Mean & 0.507 & 0.841 & 0.000 & 0.000 \\
\hline Minimum & 0.000 & 0.000 & 7.147 & 14.430 \\
\hline Maximum & 2.500 & 16.625 & 0.602 & 0.602
\end{tabular}

based calculation algorithm is correct, and the stainless steel elements nor plastic shielding of the applicator have a significant effect on the dose, which has also been reported by Lymperopoulou [32] who used Monte Carlo calculations for the same applicator but with a different TPS (based on TG-43) Plato v.14.2.7 (Nucletron, an Elekta company, Elekta AB, Stockholm, Sweden).

Our analysis of the dose distributions for applicators with tungsten shields did not provide equally good results in terms of similarities between the calculated and measured doses. The strongest disagreement of dose distribution was measured in the area closest to the applicator. This occurs because the TPS does not take into account the loss of scattered radiation due to the tungsten shielding. Therefore, since the TPS calculates the dose differently only in the shielded quarters, the dose values where the shields do not directly shield the field are counted as if there was no shield at all. Chen showed the same thing with Monte Carlo simulations [33], as did the first published survey with a thimble ionization chamber performed by Waterman and Holcomb [34]. Another factor that influenced differences in dose distributions was the shifting of the shields inside of the applicator, which was an unexpected finding when $270^{\circ}$ shielding was used. The shifting was noticed as radiation leakage between shields that left a mark on the films. As a result of this shifting, an extra dose was delivered to the area that was theoretically blocked, but also this shield movement reduced the dose in the treatment area, an area that was supposed to be unshielded according to the TPS plan. The leakage itself may be not clinically significant; the chance of occurring again in the exact same spot during subsequent radiation fractions is quite low. However, shielding of a region that is supposed to be irradiated is clearly undesirable. We were unable to measure the extent of these shifts because this was not feasible with the measuring methods used in the study.

Other reasons for the observed difference between the calculated and measured doses are related to the measurement methods. Although much research has been carried out in various aspects of dosimetry, few studies of brachytherapy dosimetry used films. The reason for this is that films are time-consuming, whereas ionizing chambers and Monte Carlo simulations are considered as faster and better tools. As a result, the lack of published data on film dosimetry in brachytherapy provided a limited foundation to prepare our study, and we only discovered areas that needed improvement after the dose comparisons. For example, we cut films with two different tools and placed the films in water; this procedure affected the final results. The quality of the edge of a film is important due to water penetration inside the film structure, and any imperfections were magnified during the digitizing procedure. In addition, small mismatches in the sizes of the holes cut to fit applicators also influenced the final result (Figure 14).

In our study, we did not used all the potential capabilities of radiochromic films. Although these films have a very high spatial resolution, the TPS permits exporting plans only in $1 \mathrm{~mm}$ resolution of the dose grids. Nevertheless, radiochromic films can be successfully used in dosimetric verifications in brachytherapy, as it is shown above. In addition, our method of placing

\section{A}
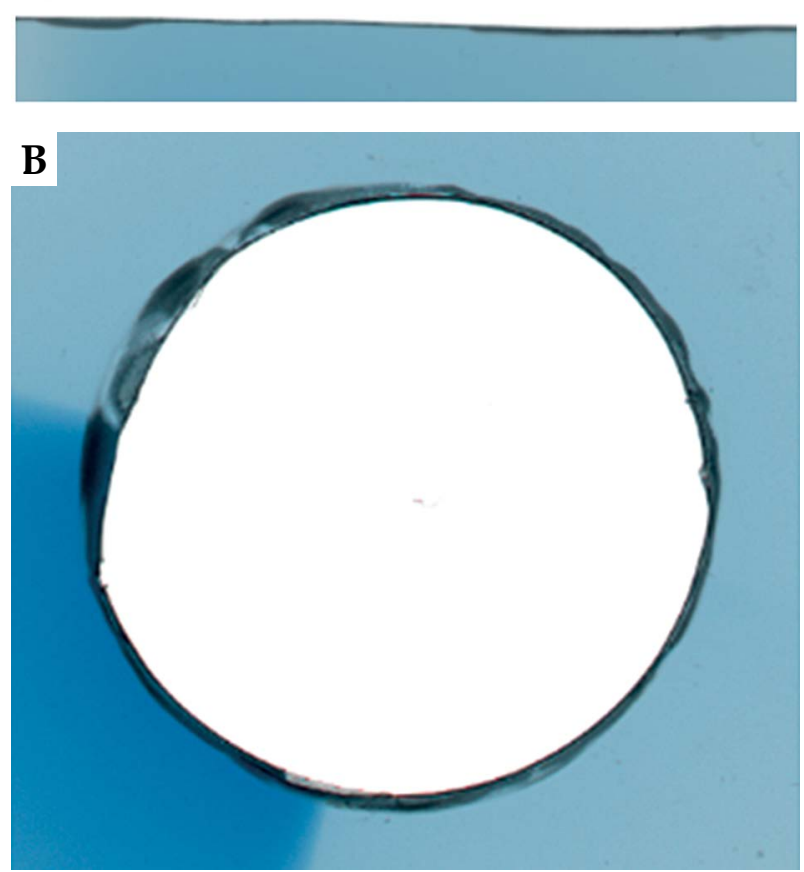

Fig. 14. Influence of cutting technique (A) lancet and (B) circle cutter on the edge of film 
the radiochromic films can be used to check the spatial arrangement of shields inside the applicator before irradiation.

Planning of the dose distribution for the cylindrical applicators used in the study, which have only one channel positioned centrally within the applicator, did not allow using sophisticated optimization algorithms. Nevertheless, for areas that do not require advanced "dose painting", this simple applicator is sufficient. In addition, for the relevant clinical cases with localized target area requiring irradiation with an intracavitary applicator, the use of shielded cylindrical applicator permits for the protection of healthy tissues.

\section{Conclusions}

Gamma analysis showed good agreement between the dose distributions calculated using TPS and the doses measured by Gafchromic films, thus showing the viability of using film dosimetry in brachytherapy.

\section{Disclosure}

Authors report no conflict of interest.

\section{References}

1. Malicki J. The importance of accurate treatment planning, delivery, and dose verification. Rep Pract Oncol Radiother 2012; 17: 63-65.

2. Palmer AL, Lee C, Ratcliffe AJ et al. Design and implementation of a film dosimetry audit tool for comparison of planned and delivered dose distributions in high dose rate (HDR) brachytherapy. Phys Med Biol 2013; 58: 6623-6640.

3. Kirisits C, Rivard MJ, Baltas D et al. Review of clinical brachytherapy uncertainties: analysis guidelines of GEC-ESTRO and the AAPM. Radiother Oncol 2014; 110: 199-212.

4. Granero D, Vijande J, Ballester F et al. Dosimetry revisited for the HDR 192Ir brachytherapy source model mHDR-v2. Med Phys 2011; 38: 487-494.

5. Alves de Oliveira C, Carmo Lopes M, Matos A. Commissioning of brachytherapy module of Oncentra MasterPlan treatment planning system. J Contemp Brachytherapy 2009; 1 : 224-230.

6. Perez-Calatayud J, Ballester F, Das RK et al. Dose calculation for photon-emitting brachytherapy sources with average energy higher than $50 \mathrm{keV}$ : Report of the AAPM and ESTRO. Med Phys 2012; 39: 2904-2929.

7. DeWerd LA, Ibbott GS, Meigooni AS. A dosimetric uncertainty analysis for photon-emitting brachytherapy sources: report of AAPM Task Group No. 138 and GEC-ESTRO. Med Phys 2011; 38: 782-801.

8. Adamczyk M, Zwierzchowski G, Malicki J et al. Evaluation of clinical benefits achievable by using different optimization algorithms during real time prostate brachytherapy. Phys Medica 2013; 29: 111-117.

9. Rivard MJ, Beaulieu L, Mourtada F. Enhancements to commissioning techniques and quality assurance of brachytherapy treatment planning systems that use model-based dose calculation algorithms. Med Phys 2010; 37: 2645-2658.

10. Hellebust TP, Kirisits C, Berger D et al. Recommendations from Gynaecological (GYN) GEC-ESTRO Working Group (III): considerations and pitfalls in commissioning and applicator reconstruction in 3D image-based treatment planning of cervix cancer brachytherapy. Radiother Oncol 2010; 96: 153-160.
11. Palmer A, Bradley D, Nisbet A. Physics-aspects of dose accuracy in high dose rate (HDR) brachytherapy: source dosimetry, treatment planning, equipment performance and in vivo verification techniques. J Contemp Brachytherapy 2012; 4: 81-91.

12. Damato AL, Cormack RA, Viswanathan AN. A novel intracavitary applicator design for the treatment of deep vaginal fornices: preliminary dose metrics and geometric analysis. J Contemp Brachytherapy 2015; 7: 48-54.

13. Hyer DE, Sheybani A, Jacobson GM et al. The dosimetric impact of heterogeneity corrections in high-dose rate (192) Ir brachytherapy for cervical cancer: investigation of both conventional Point-A and volume-optimized plans. Brachytherapy 2012; 11: 515-520.

14. Beaulieu L, Carlsson Tedgren A, Carrier JF et al. Report of the Task Group 186 on model-based dose calculation methods in brachytherapy beyond the TG-43 formalism: current status and recommendations for clinical implementation. Med Phys 2012; 39: 6208-6236.

15. Mikell JK, Klopp AH, Gonzalez GM et al. Impact of heterogeneity-based dose calculation using a deterministic grid-based Boltzmann equation solver for intracavitary brachytherapy. Int J Radiat Oncol Biol Phys 2012; 83: 417-422.

16. Oliveira SM, Teixeira NJ, Fernandes L et al. Dosimetric effect of tissue heterogeneity for $125 \mathrm{I}$ prostate implants. Rep Pract Oncol Radiother 2014; 19: 392-398.

17. Petrokokkinos L, Zourari K, Pantelis E et al. Dosimetric accuracy of a deterministic radiation transport based 192Ir brachytherapy treatment planning system. Part II: Monte Carlo and experimental verification of a multiple source dwell position plan employing a shielded applicator. Med Phys 2011; 38: 198.

18. Zourari K, Pantelis E, Moutsatsos A et al. Dosimetric accuracy of a deterministic radiation transport based 192Ir brachytherapy treatment planning system. Part III. Comparison to Monte Carlo simulation in voxelized anatomical computational models. Med Phys 2013; 40: 011712.

19. Vasiliki P, Evaggelos P, Eleftherios P et al. A user-oriented procedure for the commissioning and quality assurance testing of treatment planning system dosimetry in high-doserate brachytherapy, Brachytherapy 2016; 15: 252-262.

20. Moura ES, Micka JA, Hammer CG et al. Development of a phantom to validate high-dose-rate brachytherapy treatment planning systems with heterogeneous algorithms. Med Phys 2015; 42: 1566-1574.

21. Singh K. Investigation of the energy response of EBT-2 gafchromic film model. Department of Medical Physics, McGill University, Montreal, December 2011 - a thesis.

22. Granero D, Perez-Calatayud J, Pujades-Claumarchirant MC et al. Equivalent phantom sizes and shapes for brachytherapy dosimetric studies of 192Ir and 137Cs. Med Phys 2008; 35: 4872-4877.

23. Aldelaijan S, Mohammed H, Tomic N et al. Radiochromic film dosimetry of HDR (192) Ir source radiation fields. Med Phys 2011; 38: 6074-6083.

24. Bahadur YA, Constantinescu C, Hassouna AH et al. Single versus multichannel applicator in high-dose-rate vaginal brachytherapy optimized by inverse treatment planning. J Contemp Brachytherapy 2015; 6: 362-370.

25. Tabrizi SH, Reza Aghamiri SM, Najarian S et al. Dosimetric evaluation of a novel high dose rate (HDR) intraluminal/ interstitial brachytherapy applicator for gastrointestinal and bladder cancers. J Appl Clin Med Phys 2011; 12: 153-161.

26. Uniyal SC, Naithani UC, Sharma SD et al. Radiochromic film dosimetry of rectal inhomogeneity and applicator attenuation in high dose rate brachytherapy of uterine cervix. J Appl Clin Med Phys 2012; 13: 3654. 
27. Brown T, Hogstrom RK. Dose-response curve of EBT, EBT2, and EBT3 radiochromic films to synchrotron-produced monochromatic x-ray beams. Med Phys 2012; 39: 7412-7417.

28. Chiu-Tsao ST, Ho Y, Shankar R et al. Energy dependence of response of new high sensitivity radiochromic films for megavoltage and kilovoltage radiation energies. Med Phys 2005; 32: 3350-3354.

29. Butson MJ, Cheung T, Yu PK. Weak energy dependence of EBT gafchromic film dose response in the $50 \mathrm{kVp}-10 \mathrm{MVp}$ X-ray range. Appl Radiat Isot 2006; 64: 60-62.

30. Richter C, Pawelke J, Karsch L et al. Energy dependence of EBT-1 radiochromic film response for photon $(10 \mathrm{kVp}-15$ $\mathrm{MVp})$ and electron beams (6-18 $\mathrm{MeV})$ readout by a flatbed scanner. Med Phys 2009; 36: 5506-5514.

31. Sellakumar P, Sathish Kumar A, Sanjay S et al. Evaluation of dosimetric functions for Ir-192 source using radiochromic films. Nuclear Instruments and Methods in Physics Research Section B: Beam Interactions with Materials and Atoms 2009; 267: 1862-1866.

32. Lymperopoulou G. A Monte Carlo dosimetry study of vaginal 192Ir brachytherapy applications with a shielded cylindrical applicator set. Med Phys 2004; 31: 3080-3086.

33. Chen IJ. The Calculation of 3D Spatial Dose Distribution Around a Shielded Vaginal Cylinder with Iridium-192 Calculated by using Monte Carlo Code EGS4. Proceedings of the Second International Workshop on EGS, 8-12 August 2000, Tsukuba, Japan, KEK Proceedings 200-20, pp.107-115.

34. Waterman FM, Holcomb DE. Dose distributions produced by a shielded vaginal cylinder using a high-activity iridium-192 source. Med Phys 1994; 21: 101-106. 